\title{
P01-5-3 Poster session
}

\section{Hyperinsulinemia promotes heterologous desensitization of beta2 adrenergic receptor in airway smooth muscle in type 2 diabetes}

\author{
Qin Fu, Rui Xu
}

Department of Pharmacology, School of Basic Medicine, Tongji Medical College, Huazhong University of Science and Technology, Wuhan, China

Beta adrenergic drugs are the most common bronchodilators approved for clinical use in asthma. Obesity is reported to influence asthma severity and response to beta adrenergic receptor agonist, but the molecular mechanism underlying this correlation is unclear. Hyperinsulinemia is a hallmark of obesity/diabetes, and we show recently insulin plays a crucial role in beta-adrenergic receptor desensitization in heart. We therefore investigated whether insulin promotes beta-adrenergic receptor (beta AR) desensitization in airway smooth muscle (ASM), contributing to altered airway relaxant responsiveness to beta-agonist. Human ASM cells and freshly isolated mouse airway tissues exposed to insulin exhibit impaired beta2 AR induced cAMP accumulation and airway relaxation, respectively. The impaired relaxation responsiveness accompanying heterologous beta2 AR desensitization is associated with upregulated PDE4D phosphorylation, which is ameliorated by pretreating the ASM preparations with PDE4 inhibitor roflumilast or with inhibitor of ERK1/2 signaling. The insulin-induced upregulation of PDE4D activity is mediated through GRK2dependent beta2AR-Gi signaling for activation of ERK1/2, which promotes phosphorylation of PDE4D and PDE4D gene transcription. Furthermore, pharmacological inhibition of PDE4 effectively reverse impaired beta2AR-mediated ASM relaxation in type 2 diabetes mouse model induced by high fat diet feeding. Collectively, these findings identify that ASM beta2AR desensitization in type 2 diabetes is mediated by upregulated PDE4D phosphorylation induced by crosstalk between insulin and beta2AR signaling. Our results suggest that roflumilast may be used as a novel therapeutic agent against obesity or diabetes-associated asthma. Furthermore, GRK2-mediated signaling is also a potential therapeutic modality to prevent or treat beta2AR desensitization in ASM associated with hyperinsulinemia. 